\title{
Electric and Magnetic Energies in the Human Body
}

\author{
Salama Abd Elhady \\ Dep. of Mechanical Engineering \\ Faculty of Energy Engineering \\ Aswan University \\ Aswan, Egypt \\ salama_AbedelHady@hotmail.com
}

\begin{abstract}
The human body is actuated by electric and magnetic energies where the neural systems are found to transfer their instructions by nerve impulses in the form of electric signals or as waves of electric potential. However; the absence of a clear definition of electric charge misled the neuroscientists in recognizing the nerve impulses as electric charges and they defined them as chemical ions which contradicts their observation as waves of electric potential. According to a found definition of the electric charge as electromagnetic waves of electric potential that deletes confusions in electromagnetism, this innovative definition is found to be consistent with the neurologist's observations of the nerve impulses and its found nature as electric signals. Similarly; the neurologists succeeded in stimulating the neural system by magnetic flux through the scull without clear explanation due to the unclear definitions of the magnetic flux as electromagnetic waves of magnetic potential. However; we succeed in this article in finding plausible explanation of this stimulation by the innovative definitions of the electric charge and magnetic flux as electromagnetic waves of electric and magnetic potentials. We show the truth of these definitions by amble evidences.
\end{abstract}

Keywords: Nerve Impulses; Electric charges; Magnetic Flux, Electromagnetic waves, Thermoelectrical effects, Ionized photons.

\section{INTRODUCTION}

The electric current is traditionally defined as flow of electrons [1]. As the electric current is a flow of energy; it should have a nature of energy similar to the thermal radiation or the flow of electromagnetic waves [2]. In this article; it will be reviewed firstly how the Maxwell's wave equations were casted into an energy frame of reference to show a representation of the electric current as electromagnetic waves of electric potential [3]. Then it will be shown how one of Faraday's experiments that converted light, as electromagnetic waves, into electric current by subjecting the light to electric field may verify such innovative definition of electric current [4]. It will be shown the evidence of truth of this innovative definition of electric current as electromagnetic waves in deleting the duality confusion [5]. Such innovative definition of electric succeeded also in finding plausible explanation of one of Tesla's experiments who transmitted electric power as waves through space and explained the process of discharging electric charge from clouds during lightning $[6,7]$.

According to the traditional definition of electric current as flow of electrons, the neuroscientists were forced to define the nerve impulses as ions and, hence, their studies were limited to the electrochemical aspects of the nerve impulses [8]. However, the definition of electric charges as electromagnetic waves of electric potential is found to match the neurophysiologists' observations of the nerve impulses as a wave of electric activity [9]. According to the found matching between the observed features of the nerve impulses as waves of electric potential and the innovative definition of electric current; the nerve impulses (is) are recognized in this study as electric charges that have electric potential. Such postulate is introduced here to investigate measured thermoelectric aspects of the nerve impulses which were discarded by the definition of nerve impulses as flow of ions. Such postulate will be considered to find plausible explanation of the detected temperature difference of the neuronal cells as indication of inhomogeneous heat production and dissipation in the neuron cells in generating the electric nerve impulses [10]. It will be used also to suggest mechanisms of generation of the electric nerve impulses from stimulated rates of the biochemical reaction in the neural cells [11]. Such studies are applied to find robust solutions of found ambiguities in the neurosciences as it solved similar confusions in electromagnetism [1]).

The Nature of the Electric Charges

The definition of the nature of electric charge as energy in transfer was started by modifying the Maxwell's wave equations. The time coordinate " $t$ " in these equations was replaced by entropy "s" which is mainly a function of time and energy [13]. Such replacement casts the Maxwell's equations into an energy frame of reference which may 
express the separate flow of electric or magnetic energies [14]. The introduced frame is defined by the electric field $\mathrm{E}$, the magnetic field $\mathrm{H}$, and the entropy $\mathrm{S}$ as follows (14):

$\left(\nabla^{2}-\frac{1}{c^{2}} \frac{\partial^{2}}{s^{2}}\right) \mathrm{E}=0$

(1)

$\left(\nabla^{2}-\frac{1}{c^{2}} \frac{\partial^{2}}{s^{2}}\right) \mathrm{H}=0$,

(2)

Fig. 1 represents these equations graphically where the electric field " $E$ " is the vertical axis, the magnetic field " $\mathrm{H}$ " is the abscissa, and the entropy "s" is the ordinate [14].

Such representation succeeded in showing the flow of electric and magnetic energies during the flow an electromagnetic wave as the areas swept by the electric and the magnetic waves [15].

The flow of electric energy of zero electric potential is represented by the first integral and the magnetic energy of zero magnetic potential is represented y the second integral.

Fig. 1 depicts a graphical representation of these equations where the Electric Field " $E$ " is the vertical axis, the magnetic field " $\mathrm{H}$ " is the abscissa, and the entropy "s" is the ordinate [16]. This representation succeeds in showing the energy flow during an electromagnetic wave as the sum of the areas swept by the electric and the magnetic waves. So, the flowing electric energy per an electromagnetic wave is expressed by the first integral and the flowing magnetic energy per wave is represented also by the second integral in the following equation [16]:

$\mathrm{Q}_{\text {total }}=\mathrm{Q}_{\mathrm{el}}+\mathrm{Q}_{\mathrm{mag}}=\int_{0}^{2 \pi}|\mathrm{EdS}|+\int_{0}^{2 \pi}|\mathrm{H} \mathrm{dS}|$

Joule/wave

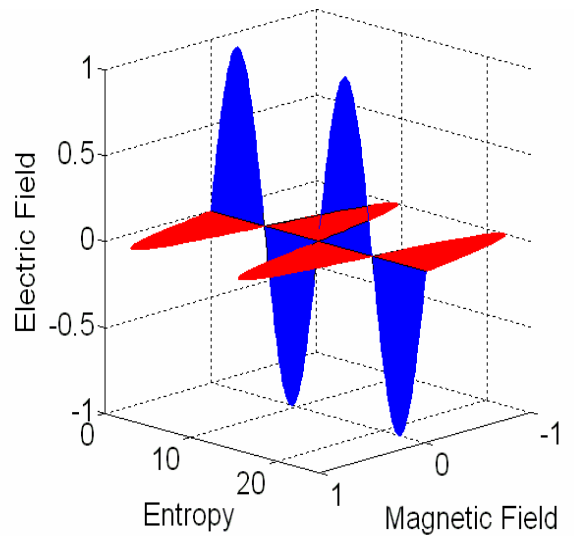

Fig. 1. Representation of flow of Electromagnetic waves in Energy frame of references composed of the energy coordinates: Electric field, Magnetic field, and Entropy, E-H-s coordinates, where the flow of the electric wave is shown in the E-s plane and the flow of the magnetic wave is shown in the $\mathrm{H}$-s plane (14).

According to previous works; it has been found that this sum which represents the quantity of energy flow per wave is equal to Planck's constant, $h$ Joule/wave [16]. Such results are confirmed by results of the early Planck's experiments [17] (Badino (2015)). Accordingly, the quantity "h v" Watt represents the rate of the energy flow of pulses, or quanta of magnitude "h," of electromagnetic waves of frequency " $v$ " [16].

In one of his experiments, Faraday succeeded in electrifying a ray of light by subjecting the ray of light to an electric field [17]. According to results of his 7experiments, he found that the magnetic field can indirectly influence the behavior of the electrified light wave as flow of electric current [18]. The influence or effect that Faraday observed is now known as Faraday's rotation because his experiment illustrated the rotation of a ray of light by the action of magnetic field, whatever the magnet's configurations are, as seen in Fig. 2 [19].

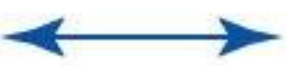

before magnetic

field

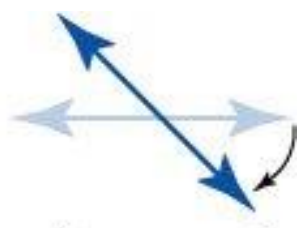
after magnetic field 
Fig. 2. Rotation of an electrified ray by a magnetic field [18]18)

The success of Faraday in converting the electromagnetic waves, or light, into electric current can be visualized as a special wave or solution of the modified Maxwell's equation. Such wave is described as a special solution of modified Maxwell's equations which have, as an initial condition, electric or positive potential of the magnitude " $+/-\Delta \overline{\mathrm{E}}$." So, the following equation represents the mathematical model of flow of electric current as electromagnetic waves of electric potential [20]:

$\mathrm{E}(\mathrm{r}, \mathrm{s})=\mathrm{E} \cos (\mathrm{kr}+\omega \mathrm{s}+\varphi)+/-\Delta \overline{\mathrm{E}}$

$\mathrm{H}(\mathrm{r}, \mathrm{s})=\mathrm{H} \cos (\mathrm{kr}+\omega \mathrm{s}+$

$\varphi)$

Equations (3) and (4) represent a solution of the differential Eqs. (1) and (2) whose initial electric field "E" is " $+/-\Delta \bar{E}$ " [20]. Such solution of the Maxwell's equations is represented graphically in Fig. 2 A and Fig. 2 B [20]. Fig. 2 A represents the electric current as a flow of negative charges in the form of electromagnetic of negative electric potential $(-\Delta \overline{\mathrm{E}})$ in a frame of energy coordinates E, H, and S. Fig. 2 B shows also the electric current as flow of positive electric charges in the form of electromagnetic waves of positive electric potential $(+\Delta \overline{\mathrm{E}})$ in a frame of energy coordinates $\mathrm{E}, \mathrm{H}$, and $\mathrm{S}$. Both figures represents visualization of the electric current as electromagnetic or energy waves whose frequency is " $\mathrm{v}$ " and whose electric potential is " $+/-\Delta \overline{\mathrm{E}}$." The potential of the wave is determined by the location of the axis of its oscillations. So, the electric charge may be defined as ionized quanta of energy, or photon, of potential “+/- $\Delta \overline{\mathrm{E}}$ “ $[20]$.

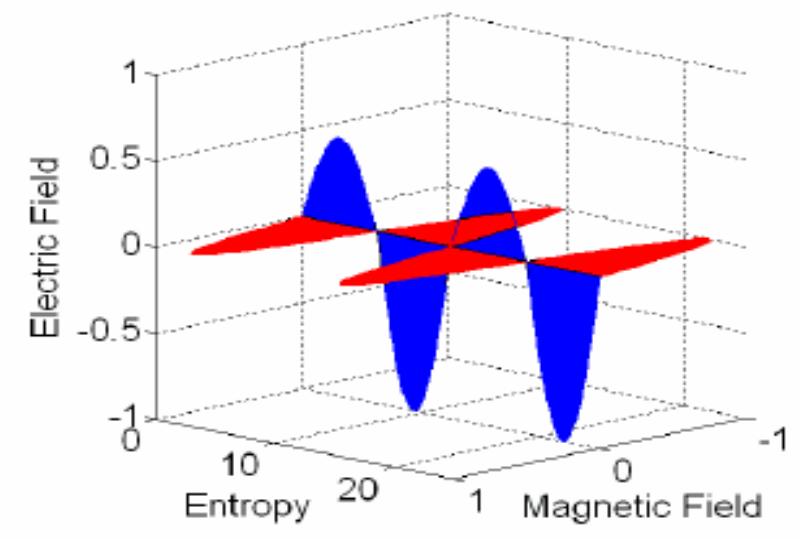

Fig. 3. A. Flow of negative electric charges as flow of electromagnetic waves of -ve potential where the electric energy in the E-s plane is oscillating around the negative potential " $-\Delta \bar{E}$ " [20].

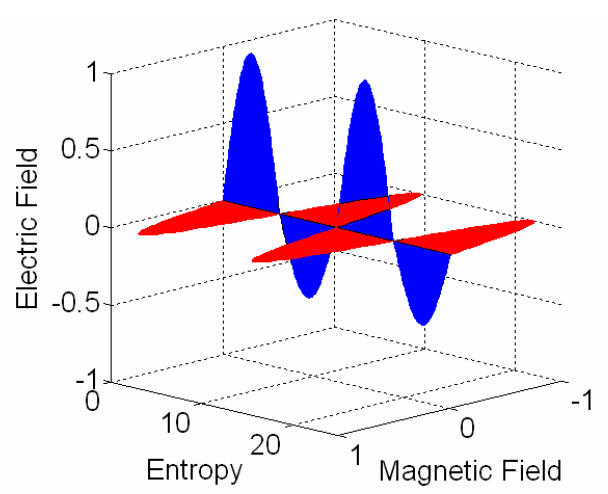

Fig. 3. B. Flow of negative electric charges as flow of electromagnetic waves of -ve potential where the electric energy in the E-s plane is oscillating around the negative potential " $+\Delta \overline{\mathrm{E}}$ " [20].

So, electrifying a ray is interpreted by innovative definition of electric current as converting the electromagnetic waves into electric current when gaining electric potential during the electrification process. This was the first definitive evidence that light and electromagnetism are related, and paved the way for Maxwell's brilliant theoretical demonstration of the definition of thermal radiation as electromagnetic waves, and their identity with light [21]. However; the Tesla's discovery of "Radiant energy" was also interpreted as normal radiation of electric energy or as electromagnetic waves of electric potential from the Tesla's tower [22]. Tesla's experiment is found similar to the discharge of (accumulation of) accumulated electric charges as electrified electromagnetic 
waves from clouds during the lightening [23]. Both results prove also the truth of the postulated definition of electric current as electromagnetic waves of electric potential [24].

Similarly; the magnetic flux can be represented graphically and analytically as electromagnetic wave of magnetic potential by Fig. (4) and the following equations [25]:

$\mathrm{E}(\mathrm{r}, \mathrm{s})=g_{1}(\omega \mathrm{s}-\mathrm{kr})$

$\mathrm{H}(\mathrm{r}, \mathrm{s})=g_{2}(\omega \mathrm{s}-\mathrm{kr})+/-\Delta \mathrm{H}$

Graphical representation of this equation is shown in Fig. 4.

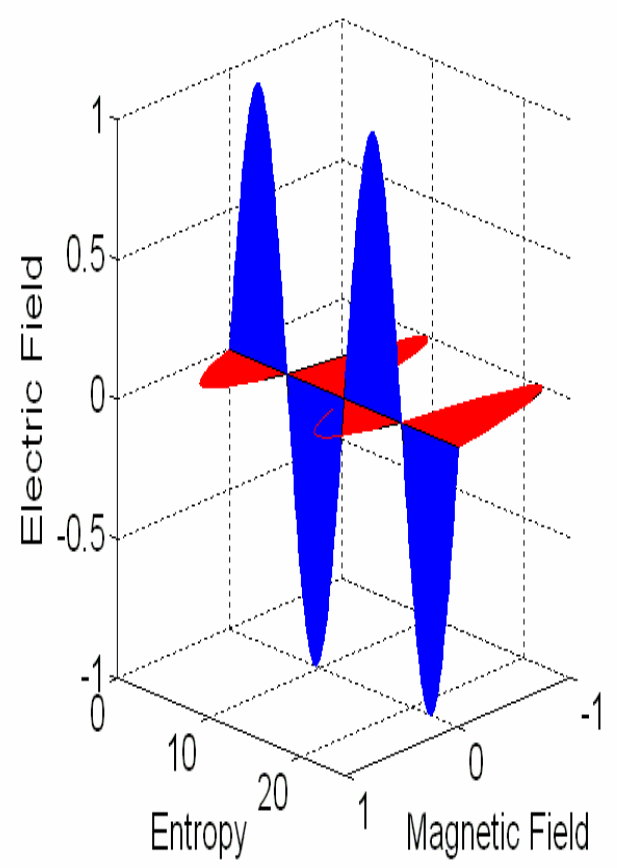

Fig.4: Graphical representation of magnetic flux [14].

Such definitions of electric current and magnetic flux succeeded in deleting the "duality confusion" and represent clear definitions of the nature of electric charge and magnetic flux that researchers may not found in literature (5). The success of research teams and technologists in pumping electric energy through optical fiber system represents a proof of the nature of electric current as waves that may flow also through nerves [26].

\section{Nature of the Nerve Impulses}

According to Hodgkin and Huxley, nerve impulse consists of a rapid and coordinated sodium ion influx and the subsequent potassium ions exit through the membrane of the excitable cells [27]. Such model of Hodgkin and Huxley has been valued for the past sixty years as a foundation for neuroscientific research. Over the years, however, a number of scholars have challenged the completeness of the conventional $\mathrm{HH}$ model for various reasons, in particular its incapability to account for thermoelectric aspects of the nerve impulse as its definition as ions limits the study to its electrochemical aspects [28]. Several alternative models have been proposed that do take into account the non-electrical aspects of the nerve impulse and emphasize their importance in gaining a more complete understanding of the nature of the nerve impulse. For instance, considerable evidence indicates that action potentials are accompanied by production and subsequent absorption of heat that are of non-electrical nature [29]. However; most of the studies were adhered to the definition of electric current as flow of electrons and the definition of the electric charge, which should have an energy nature, as electrons. So, they consider the nerve impulse just as flow of ions [30].

However, the observations of the neuroscientists found the flow of nerve impulses in general as a wave of electric activity or a measured 'action potential' [31]. Similarly, the observed nature of nerve impulse as a wave of electric activity is directly consistent with the innovative definition of electric current as flow of electromagnetic waves of electric potential (14). So, the flow of nerve impulses can be recognized according to such definition of electric current directly as flow of electric charges or as electromagnetic waves of electric potential. Such identification may account for found phenomena that accompany nerve impulse propagation for which there is nevertheless ample evidence [32]. Additionally; measurement results of temperature in the neural system detected temperature difference between the cell body and neurites, thus the identification of the nerve impulse as ions cannot afford a plausible explanation for such detection (6). Similarly, the dependence of the neurosciences on the definition 
of electric current as flow of electrons, which is illogic, is a source of the found confusions in the field of neurosciences according to many references [33].

The description of flow of nerve impulses as electric current is also featured by the use of patch clamp technique that allowed measurement of current-voltage characteristics of the cell's membrane [33, 34]. According to the measurements of Goldman, Hodgkin, and Katz (GHK), we get the description of fully activated current-voltage characteristics of a patch from the cell membrane that indicate the possibility of flow of the nerve impulses as electric charges or the ability of the cell membrane to conduct the nerve impulses as electric current $[35,36]$.

\section{Thermoelectrical Aspects of The Electric Current}

Thermoelectricity is concerned by coupled transport of heat and electricity. Such phenomena are mainly characterized by the Seebeck effect which is defined as follows [37]:

$$
S=-\frac{\Delta V}{\Delta T}
$$

This coefficient relates the conversion of the thermal potential of the incident heat $\Delta T$, into electric potential $\Delta V$. Thus it characterizes the conversion of the thermal radiation of thermal potential $\Delta T$, into electric current of electric potential $\Delta V$ [37].

By the innovative definition of electric current as flow of electromagnetic waves of electric potential, it was possible to define the PV effect as the conversion of the incident radiation or heat into electric current when crossing junctions of materials of different Seebeck coefficients or of different charge carriers [38]. Such definition is verified in all thermoelectric devices as thermocouples or thermopiles, thermoelectric generators, and photovoltaic cells [39]. In case of photovoltaic cells, the incident heat passes through a junction separating a layer of higher number of electrons, $\mathrm{n}$-type side, and a layer of higher number of holes, p-type side, Fig. 4 [40]. So, the thermal potential of the incident radiation that forces the heat to flow across the PV cell is converted into electric potential when it crosses the p-n junction of the cell. Such electric potential is proportional to the thermal potential of the incident radiation by Seebeck coefficient, as constant of proportionality, according to Eq. (5) [41].

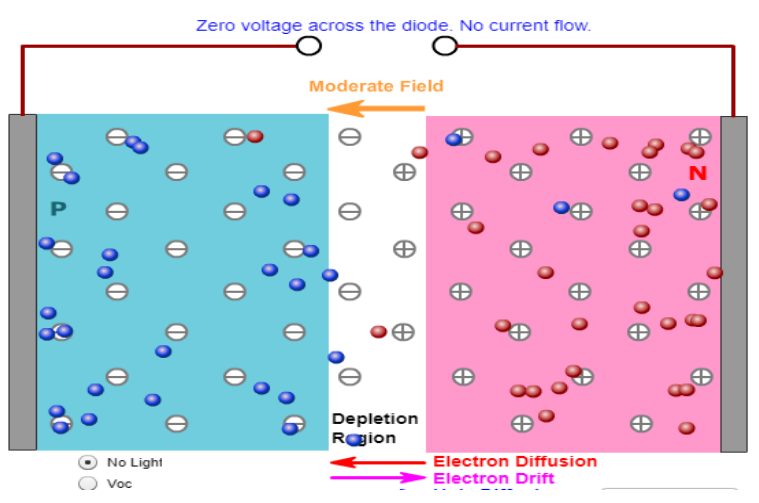

Fig. 5. Movement of charge carriers in the Photovoltaic Cell, [40].

3. Thermoelectric Aspects of the Electric Nerve Impulses

Recognizing the nature of nerve impulses as electric charges, it is possible to investigate thermoelectrical aspect of these impulses where its definition as "Ionic Current" limited our investigation to its electrochemical aspects only [42]. Such limitation represents a barrier to investigate the source of the nerve impulses in the neural system and the detected intracellular temperature differences that have recently been found [43]. However; the Soliton model and other neural models tried to study the found thermoelectric aspects of the nerve impulses but they were adhered to their definition as ions which limited their studies to the electrochemical aspects of the nerve impulses only [44].

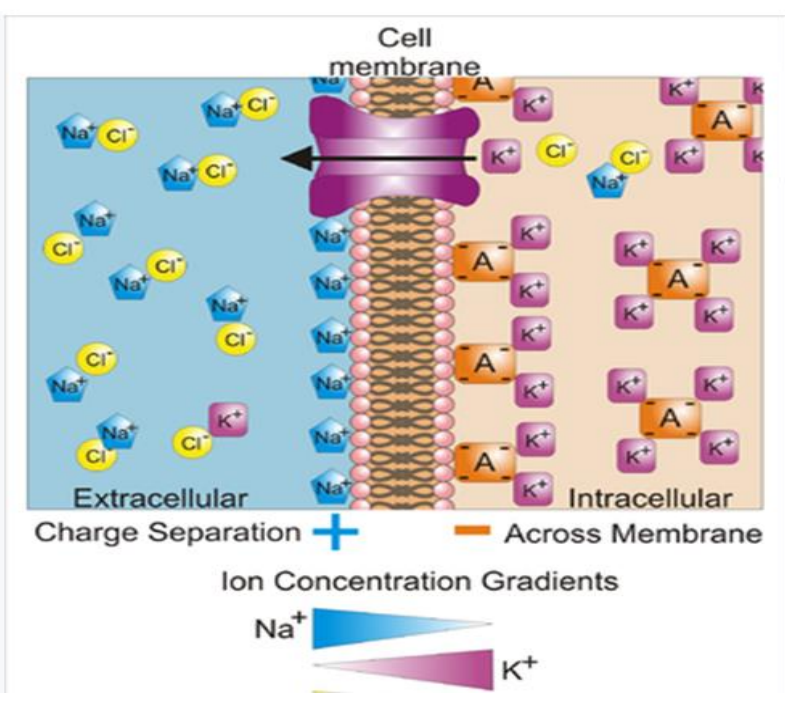


Fig. 6. Differences in the concentrations of ions on opposite sides of a cellular

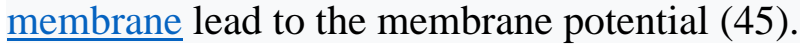

Measurement results of temperature in the neural system show that the temperatures in the cell body and neurites are different and thus suggest inhomogeneous heat production and dissipation in the cells [6]. So, it is possible also to suggest a change in the rate of the biochemical exothermic reactions in the stimulated cells as a response to received stimuli. According to the neural investigations, there is found difference in the concentrations of ions on the both sides of the cell membrane that leads to measure a membrane potential in the range $-40 \mathrm{mV}$ to $-70 \mathrm{mV}$ [45]. So; identifying the similarity of distribution of different charge carriers on the two sides of a photovoltaic cell, Fig. 5, and the distributions of different ions on the sides of the cell membrane, Fig.6, suggest the conversion of the heat flow from the cells across the cell membrane into electric current by similar mechanism of conversion of the heat flow across the PV cell into electric current by Seebeck effect. The evidence of the truth of this postulate is verified by the measured flow of wave impulses across the cell membrane in the form of waves of electric potential [46]. According to neurosciences; this flow is distinguished as a standalone electric potential while the nature of the nerve impulse should be defined as energy driven by electric potential since it is capable of motivating another cells [47, 48]. So; it is essential to nominate such action potential as the driving potential of electric energy that flow across the cell membrane. Fig. 7 shows the measured wave where the abscissa is the electric potential of the nerve impulse while its ordinate was considered as time. According to the definition of entropy as a measure of time; it is possible to replace the time scale " $\mathrm{t}$ " in the ordinate by entropy scale "s" [48]. So, we get a representation of the nerve wave in an energy frame of reference defined by the E-s coordinate as we have casted the Maxwell's wave equation in a similar frame of reference [14]. So, the energy of a nerve impulse can be estimated in such frame by the area enclosed by the wave and the entropycoordinate as follows:

$Q=\int E d S$

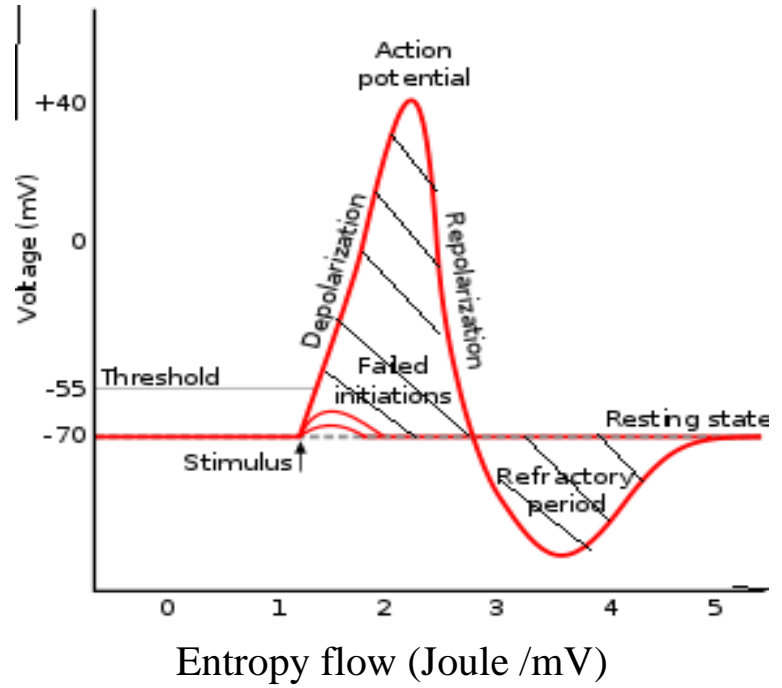

Fig. 7. Representation of the nerve impulse in E-s energy coordinates

According to this integral; the energy of nerve impulse is actually represented by the shaded area in Fig. 7 [49]. The source of this energy is the biochemical exothermic reactions inside the cells which are converted into electric energy when crossing the cell membrane by Seebeck effect [47]. In this case; the response of the cell to stimuli is performed through increase of its temperature by increase of the rate of the exothermic reactions in this cell. This suggested relation between the Seebeck effect and the neural system was found by Seebeck, Volta, and Galvani who observed that electrical stimulation caused a dead frog's leg to twitch during their discovery of the Seebeck effect that converted the temperature difference into electric potential, or action potential $[48,49,50]$.

According to literature; the brain is the source of transmitted power, information and instructions to the neurons of the body and it has higher temperature than the body and it consumes $20 \%$ of the body's oxygen and energy [51]. Such data represents an evidence to conclude that nerve impulses are produced by uniform biochemical reactions that consume energy and oxygen in the brain cells and it is not just electrochemical processes producing a standalone electric potential without motivating energy [52].

4. Influence of the magnetic flux on the Scull 


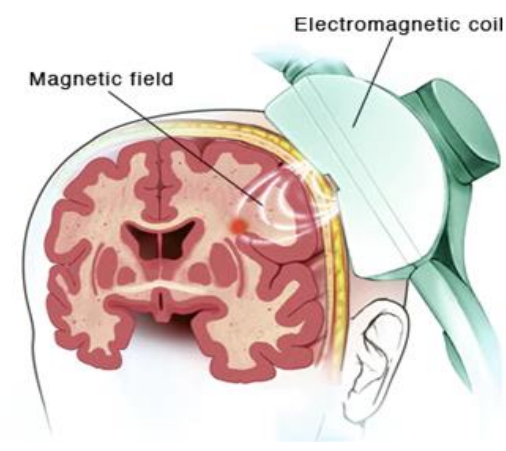

Fig. 8. Repetitive Transcranial Magnetic Stimulation (rTMS)

The definitions of magnetic flux and electric charge as electromagnetic waves of electric or magnetic potential may help to understand Transcranial magnetic stimulation (TMS) [53, 54]. Such noninvasive procedure uses magnetic fields to stimulate nerve cells in the brain, as seen in Fig. 8 [54], to improve symptoms of depression. TMS is typically used when other depression treatments haven't been effective. This treatment for depression involves delivering repetitive magnetic pulses, so it's called repetitive TMS or rTMS. Sending the magnetic flux to the cells membrane of the inert neurons in the brain; such flux may represents an energy shock that heats up suddenly the cells of the brain. Then the membrane will perform its function to convert the produced heat into nerve impulses or electric signals by converting its thermal potential into electric potential. In this way; regions in the brain are activated and is capable to send nerve impulses or electric signals to motivate the body to desirable changes.

\section{Conclusions}

According to an innovative definition of the electric current as flow of electromagnetic waves of electric potential; it was possible to recognize the nature of the nerve impulses directly as electric charges that have electric potential. Such recognized nature allows the study of thermoelectric aspects of the nerve impulses and finds plausible explanation of the detected temperature differences between the cell body and neurites. Accordingly; it is suggested similar mechanisms of converting the heat produced by the biochemical exothermic reactions in the cells into electric nerve impulses similar to the mechanism that converts the heat incident on the PV cells into electric current by the Seebeck effect. The truth of postulating the nerve impulse as electric charges and the conversion of the heat flow across the cell membrane into electric nerve impulses are supported by ample evidences.

\section{References}

1. Jewett, Jr. and R. A. Serway, "Physics for Scientists and Engineers with Modern Physics," 7th Edition, Thomson, 2008

2. N. J. Carron, "On the Field of a Torque and the Observability of the Vector Potential," Physics Notes, Note 6, 1993.

3. Abdelhady, S. A Fundamental Equation of thermodynamics that Embraces Electrical and Magnetic Potentials, J. Electromagnetic Analysis \& Applications , 2, 162- 166. (2010.)

4. Faraday, M.. Experimental Researches in Electricity, Nineteenth Series, Phil. Trans. R. Soc. Lond, 136, 1-20, doi: 10.1098/rstl.1846.0001. (1846)

5. Abdelhady, S., Innovative Understanding of the Duality confusion, the Photovoltaic and the Magnetocaloric Effects. Ain Shams Engineering Journal, Engineering Physics and Mathematics, 9, 2283-2289. (2018.).

6. Tesla, N., The effect of static on wireless transmission, Electrical Experimenter, 627-658.( 1919).

7. Karthick, S., Jason, G. Lightning as Atmospheric Electricity Conference Paper in Canadian Conference on Electrical and Computer Engineering DOI:10.1109/CCECE.2006.277659. Source: IEEE Xplore. (2006).

8. Hodgkin, A. L., and Huxley, A. F. A quantitative description of membrane current and its application to conduction and excitation in nerve. J. physiol. 117, 500-544. (1952).

9. Benjamin, D, Hanna A. H., Martin, V., Jeroen, J. G., Pieter, V., Gerrit, G., Henk, W. R.,. Thinking about the nerve impulse: A critical analysis of the electricity centered conception of nerve excitability, Progress in Neurobiology. 169, 172-185.( 2018).

10. Ryuichi, T., et. al., Detection of Temperature Difference in Neuronal Cells, Nature, Scientific Reports, 6:22071, 1-10, | DOI: 10.1038/srep22071 (2016)

11. Ryuichi, T., et. al., Detection of Temperature Difference in Neuronal Cells, Nature, Scientific Reports, 6:22071, 1-10, |DOI: 10.1038/srep22071 (2016)

12. Emilia, B., et. al, A microfluidic platform for controlled biochemical stimulation of twin neuronal networks, Biomicrofluidics, 6, 024106, 1-10, (2012)

13. Abdelhady, S., Cheng, C. H.. Advanced Thermodynamics Engineering, Scitus Academics; 1 st Edition, New York (USA) ISBN: 978168117. (2019).

14. Abdelhady, S. An Advanced Review of thermodynamics of Electromagnetism," International Journal 
of Research studies in Science, Engineering and Technology, 3, 10 - 19.( 2015.)

15. Abdelhady, S., Review of Thermodynamics of Systems that Embrace Transfer of Electric and Magnetic Energies, Journal of Physical Science and Application, 8, 112, doi: 10.17265/2159-5348/2018.01.001, 8. (2018)

16. Fraday, M., Experimental Researches in Chemistry and Physics. London: Richard Taylor and William Francis. pp. 33-53. ISBN 978-0-85066-841-4,(1895).

17. Williams, L. P., Michael Faraday: A Biography. New York: Basic Books. pp. 122-123. ISBN 978-0-30680299-7, (1965).

18. Jenkins, A., Michael Faraday's Mental Exercises: An Artisan Essay-Circle in Regency London. Oxford University Press, (2008)..

19. Abdelhady, S., Abdelhady, M. S.,. An entropy approach to the natures of the electric charge and magnetic flux, Journal of Electromagnetic Analysis \&Applications, 7, 265 - 275.( 2015)

20. Abdelhady, S., An Advanced Review of thermodynamics of Electromagnetism," International Journal of Research studies in Science, Engineering and Technology, 3, $10-19$. (2015).

21. David L. Anderson, The Discovery of the Electron (Princeton, N.J.: Van Nostrand, (1964)

22. Abdelhady, S. An Entropy Approach to Tesla's discovery of Wireless Power Transmission. Journal of Electromagnetic Analysis \& Applications, 5, 157-161.( 2013.).

23. Gosline, A., "Thunderbolts from Space," New Scientist, 186, 2498, 30-34, (2005).

24. Abdelhady, S. et al., Thermodynamics: Fundamentals and its Application in Science, Auris Reference; 1st edition, London (UK), ISBN-10: 1788020626 An International Text Book In Science.( 2017).

25. Abdelhady, S. Salama Abdelhady, Thermodynamic Analysis of Electric Charges and Magnetic Flux, Cairo 11th International Conference on Energy and Environment, Ghurgada, Egypt, 175-185.( 2009.).

26. Matsuura, M., Sato, J., Furugori, H. $60 \mathrm{~W}$ powerover-fiber feed using double-clad fibers for radio-over-fiber systems with optically powered remote antenna units, Optics Letters, 40, 5598-5601. DOI: 10.1364/OL.40.005598.( .2015).

27. Bullock, T. H., Orkand, R., and Grinnell, A., Introduction to Nervous Systems. San Francisco, CA: W. H. Freeman and Co., 559. (1977).

28. Ritchie, J. M., and Keynes, R. D. The production and absorption of heat associated with electrical activity in nerve and electric organ. Q. Rev. Biophys. 18, 451-476. doi: 10.1017/S0033583500005382. (1985)

29. Tasaki, I. and Byrne, P. M.: Heat production associated with a propagated impulse in bullfrog myelinated nerve fibers, Jpn. J.Physiol., 42, 805-813, (1992).

30. Mireille, L., Allaman, I, Magistretti, P. Brain Energy Metabolism: Focus on Astrocyte-Neuron Metabolic
Cooperation, Cell Metabolism, 14, 724-738, https://doi.org/10.1016/j.cmet.2011.08.016.( 2011).

31. Andrew, S., Johnson, W. W., The Soliton and the Action Potential- Primary Elements Underlying Sentience, Frontiers in Physiology 9, 779 - 784. doi: 10.3389/fphys.2018.00779, 1-10.( 2018).

32. Rossi, S., Roncati, E.Z., Mauri, I., Columbo, A., Stocchetti, N., Brain temperature, body core temperature, and intracranial pressure in acute cerebral damage., J Neurol Neurosurg Psychiatry;71:448-454, (2001).

33. Sakmann, B.; Neher, E., "Patch clamp techniques for studying ionic channels in excitable membranes". Annual Review of Physiology. 46: 455-472. doi:10.1146/annurev.ph.46.030184.002323, . (1984).

34. Hamill OP, Marty A, Neher E, Sakmann B, Sigworth FJ.; Marty; Neher; Sakmann; S., "Improved patch-clamp techniques for high-resolution current recording from cells and cell-free membrane patches". Pflügers Archiv: European Journal of Physiology. 391 (2): 85-100. CiteSeerX 10.1.1.456.107. doi:10.1007/BF00656997 (1981).

35. Philip, J. W,. et al., Advanced patch-clamp techniques and single-channel analysis, Journal of Experimental Botany, Vol. 50, Special Issue, pp. 103710541(1999).

36. Clay, J. R., Novel description of ionic currents recorded with the action potential clamp technique: application to excitatory currents in suprachiasmatic nucleus neurons, Neurophysio, 114: 707-716, (2015).

37. Riffat, S. B, Ma, X.,.Thermoelectrics: A review of present and potential applications," Appl Therm Eng, 23: 913-935. (2003

38. Sadiku, Matthew N. O. Elements of Electromagnetics (4th ed.). Oxford University Press. ISBN 019-5300483Ertt.( 2006).

39. Abdelhady, S. Advanced Physics of Thermoelectric Generators and Photovoltaic Cells, American Journal of Physics and Applications, 6, 133-141, journal id=622\& doi10.11648/j.ajpa.20180605.14.( 2018).

40. Wurfel, U., Cuevas, A., Charge Carrier Separation in Solar Cells, IEEE Journal of Photovoltaics, 5, 1, 461469,(2015)

41. Van Herwaarden, A. W., Sarro, P. M., Thermal Sensors Based on The Seebeck Effect, Sensors and Actuators,, 10, 321-346.(1986).

42. Appali, R., Van Rienen, U., Heimburg, T.. A comparison of the Hodgkin-Huxley model and the soliton theory for the action potential in nerves, Advances in Planar Lipid Bilayers and Liposomes, 16, 275-299. doi: 10.1016/B978-0-12-396534-9.00 009-X.( 2012).

43. Clay,J. R. Determining $\mathrm{K}+$ channel activation curves from $\mathrm{K}+$ channel currents often requires the GoldmanHodgin-Katz equation, Frontiers in Cellular Neurosciences, 3 , 1-6, (2009).

44. Hille B, Catterall WA. " Electrical Excitability and Ion Channels". Basic neurochemistry: molecular, cellular, and medical aspects. Philadelphia: Lippincott-Raven. ISBN 9780-397-51820-3. (1999). 
45. Mummert H, Gradmann D., "Action potentials in Acetabularia: measurement and simulation of voltage-gated fluxes". Journal of Membrane Biology. 124 (3): 265-73. doi:10.1007/BF01994359. PMID 1664861, (1991).

46. Goldin AL.,"Neuronal Channels and Receptors". In Waxman SG (ed.). Molecular Neurology. Burlington, MA: Elsevier Academic Press. pp. 43-58. ISBN 978-0-12-3695093, (1991).

47. Chapman RF., "Nervous system". The insects: structure and function. Cambridge University Press. pp. 533568. ISBN 978-0-521-57890(1984).

48. Ross G. Grand unified theories. Westview Press;

49. Hormuzdi SG, Filippov MA, Mitropoulou G, et al., Electrical synapses: a dynamic signaling system that shapes the activity of neuronal networks. Biochim. Biophys. Acta 1662 (1-2): 113-37. doi:10.1016/j.bbamem.2003.10.023. PMID: 15033583, (2004).

50. Raichle, M.E., Two views of brain function. Trends Cogn. Sci. 14, 180-190 (2010).

51. Allaman, I., Magistretti, P. J. Brain energy metabolism. In fundamental Neuroscience, San Diego Academic Press (2013)

52. Attwell, D., Laughlin, S. B., An energy Budget for signaling in the grey matter of the brain. Blood Flow Matab. 21. 1133-1145 (2001)

53. Khedr, E.M., Farweez, H.M., and Islam, H.. Therapeutic effect of repetitive transcranial magnetic stimulation on motor function in Parkinson's disease patients. Eur. J. Neurol. 10, 567-572 (2003)

54. Khedr, E.M., Ahmed, M.A., Fathy, N., and Rothwell, J.C. (2005a). Therapeutic trial of repetitive transcranial magnetic stimulation after acute ischemic stroke. Neurology 65, 466-468 (2005).

Allaman, I., Magistretti, P. J. Brain energy metabolism. In fundamental Neuroscience, San Diego Academic Press (2013)

Attwell, D., Laughlin, S. B., An energy Budget for signaling in the grey matter of the brain. Blood Flow Matab. 21. 1133-1145 (2001) 